\title{
Bibliotecaescolarparalasociedaddel conodimientoenEspaña
}

Miguel García-Quismondo

Correo electrónico: mmarzal@bib.uc3m.es

AuroraCuevas Cerveró

Correo electrónico: accerver@bib.uc3m.es

\section{Resumen}

El objetivo del artículo se dirige a describir la transformación de las bibliotecas escolares en CREA (Centros de Recursos para la Enseñanza y Aprendizaje), como modo de cumplir una función eficaz en la Educación para la Sociedad del Conocimiento. El estudio se realiza en el caso español, por lo que se aborda el desarrollo legislativo para comprender la situación actual de las bibliotecas escolares en España, como un estado del arte, para presentar luego el modelo teórico, la definición y aspectos organizativos del CREA. El gran reto es la conversión de la biblioteca escolar en una biblioteca digital educativa, por lo que el artículo analiza las disfunciones de la biblioteca escolar en los nuevos entornos tecnológicos, los problemas que se plantean en la transformación, los elementos sobre los que descansará la transformación, para finalmente presentar una propuesta de los instrumentos actualmente existentes y eficaces para la gestión de contenidos digitales educativos y la organización del conocimiento en la biblioteca digital educativa que será el CREA.

Palabras clave

Biblioteca escolar. Centros de recursos para la enseñanza y aprendizaje. Gestión de contenidos digitales educativos. Biblioteca digital educativa.

\section{School library for the knowledge society in Spain}

\section{Abstract \\ The main goal of this article is to describe the transformation of school libraries into CREA (Spanish term for Teaching \& Learning Resources Centres) as a requirement for them to fulfil an effective function in Education for the Knowledge Society. Approaching the Spanish case, it starts analysing the Spanish legislative framework as a means to understand the current situation of school libraries in the country. Then, it presents a theoretical model, a definition and managerial aspects related to CREA. Considering the conversion of school libraries into educational digital libraries as the biggest challenge, the article also analyses the malfunctions experienced by school libraries in technological environments, the challenges for their transformation and the elements upon which such transformation will be built. It finally presents existing and possible instruments for managing educational digital contents as well as for organizing knowledge in next generation CREA educational digital libraries.}

Keywords

School libraries. Teaching \& learning resources centres. Educational digital contents management. Educational digital library.

\section{INTRODUCCIÓN: REFORMA EDUCATIVA Y BIBLIOTECAESCOLAR}

\section{Desarrollolegislativo.Pautasgenerales}

El punto departida queestablecelas pautas generales que han de orientar el desarrollo normativo internacional lo encontramos en la obra deF. L. Carroll yP.F.Beilke, publicadoen 1979*, Guidelinesfor theplanning and organization of School Library Media Centers, primer documento en el que se establecían las líneas básicas para crear una BE. Unos años más tarde una nueva publicaciónsobreel temasirvióparaampliarycompletar la obrainicial, Guidelines for school libraries, ${ }^{* *}$ en esta ocasión se especificaban los elementos materiales, personalesyorganizativosquehan deintegrarel sistema bibliotecario escolar deun país. Hannesdóttir publica un nuevo documento en $1995^{\text {*** }}$ en el queseenmarcan las Bibliotecas Escolaresen laSociedad delaInformación y se define el nuevo perfil del bibliotecario escolar en estecontexto.

Enel año2000 sepublioóel documento IFLA/UNESCO SchoolL ibraryManifesto: TheSchool L ibraryinTeachingand Learning for All. ${ }^{* * * *}$ Lapublicación fuemuybien acogida internacionalmente y se ha traducido a diferentes idiomas, detal forma que bibliotecarios de distintas partes del mundo están utilizando estedocumento para promoverlaBEensuscentroseducativos. School Library Guidelines, plenamenteintegrada en la Sociedad delaInformación, establecen lamisión y funciones deestas bibliotecas, los recursos yel perfil del profesional bibliotecario escolar,

${ }^{*}$ CARROLL, F. L. y BEILKE, P. F. 1979. Guidelines for the planning and organization of School Library Media Centers. Paris: UNESCO ${ }^{* *}$ CARROLL, F. 1990. Guidelines for school libraries. The Hague: IFLA. *** IFLA/UNESCO. 2000. IFLA/ UNESCO School Library Manifesto: TheSchool Library in Teaching and Learning for All. [en línea]. Disponible en: <http:// www.ifla.org/VII/ s11/pubs/ manifest.htm>. [Consulta: 91-2007].

**ak* FLA/UNESCO. 2002. School Library Guidelines. [enlínea]. Disponible en: <http:// www.ifla.org/VII/s11/pubs/ sguide02.pdf>. [Consulta: 91-2007]. Puede consultarse el documento también en español. Disponibleen: <http:// www.ifla.org/VII/s11/pubs/ sguide02-s.pdf>. [Consulta: 14-1-2007].

Ley 16/1985 del 25 dejunio de 1985. BOE de 29 de junio de 1985. Disponible en: <http:/ / www.fundaciongsr.es/ documentos/leyes/ leyphe.htm >. [Consulta: 14-1-2007].es el texto legal de mayor rango, tras la constitución, que regula las bibliotecas españolas. 
losprogramasyactividadesquehan dedesarrollarse, la promoción delabibliotecaydel aprendizaje, laformación deusuariosylaAlfabetización en Información.

\section{DesarmollolegislativoenEspaña}

La Ley del Patrimonio Histórico Español ${ }^{*}$ en su título VII, definelasBibliotecas, archivosymuseos. El desarrollo deestetítulo diolugaral Real Decreto 582/1989, ${ }^{* *}$ porel queseapruebael ReglamentodeBibliotecasPúblicas del Estado y del Sistema Español deBibliotecas. En este reglamento quedan excluidaslas Bibliotecas Escolares, un antecedente claro del escaso tratamiento queestas Bibliotecasvan a recibir en lalegislación española.

En el Anteproyecto de una nueva Ley Orgánica de Educación, propuesto por el cambiodegobierno parael debatecon los sectoresimplicados, en el artículo 108, del título IV, capítuloII, sededican cuatro puntosalaBE que, porsubrevedad, transcribimosa continuación ${ }^{* * *}$ :

1. Los centros públicos dispondrán de una biblioteca escolar, cuya dotación de recursos se hará de manera progresiva por las Administraciones educativas correspondientes.

2. Las bibliotecas escolares contribuirán aqueel alumno acceda a la información y otros recursos para el aprendizajey pueda formarse en el uso crítico delos mismos.

3. La organización delas bibliotecas escolares deberá permitir quefuncionen como un espacio abierto a la comunidad educativa delos centros respectivos.

4. Los centros podrán suscribir convenios con los municipios respectivos, para el uso de bibliotecas municipalesconlasfinalidadesprevistasen esteartículo.

FinalmentelaleyOrgánicadeEducación(LOE), demayo de2006, estableceen el título preliminary capítulo 13 respectivamente.

“Los poderes públicos prestarán una atención prioritariaal conjunto defactores quefavorecen la calidaddelaenseñanzay, en especial, lacualificación

* REAL DECRETO 582/ 1989, de 19 de mayo, BOE del 31 de mayo de 1989. Disponible en: <http:// travesia.mcu.es/documentos/ rd_seb.pdf>. [Consulta: 14-1-2007].

${ }^{*}$ ĀNTEPROYECTO DE LEY ORGÁNICA DE EDUCACIÓN. Disponible en: <http:// www.mec.es/files/Anteproyecto_Ley_ Organica Educacion.pdf>. [Consulta: 9-1-2007]

${ }^{* * *}$ LEY OR̄GÁNICA DE EDUCACIÓN (LOE) 2/ 2006, de 3 de Mayo, BOE 106, de4 deMayo de2006. Disponibleen: 〈http:/ / www.mec.es/ meod/gabipren/ documentos/A17158-17207.pdf>. [Consulta: 14-1-07]. yformación del profesorado, su trabajo en equipo, la dotación derecursos educativos, lainvestigación, la experimentación y la renovación educativa, el fomento de la lectura y el uso de bibliotecas, la autonomía pedagógica, organizativaydegestión, la función directiva, la orientación educativa y profesional, lainspeoción educativayla evaluación."

“1. Los centros de enseñanza dispondrán de una bibliotecaesoolar.

2. Las Administraciones educativas completarán la dotación delas bibliotecas delos centros públicos de forma progresiva. A tal fin elaborarán un plan que permita alcanzar dicho objetivo dentro del periodo deimplantación delapresenteLey.

3. Las bibliotecas escolares contribuirán a fomentar lalecturayaqueel alumno aocedaalainformacióny otrosrecursosparael aprendizajedelasdemásáreasy materias y pueda formarse en el uso crítico delos mismos. Igualmente, contribuirán a hacer efectivolo dispuesto en los artículos 19.3y 26.2 dela presente Ley.

4. Laorganización delas bibliotecas escolaresdeberá permitirquefuncionen como un espacio abiertoala comunidad educativa delos centros respectivos.

5. Los centros podrán llegar a acuerdos con los municipios respectivos, para el uso de bibliotecas municipales con las finalidades previstas en este artículo."

Esta regulación, evidentemente, es positiva*, pues instaura la obligatoriedad de la BE en los Centros Educativos públicos* ${ }^{* *}$ por primera vez, pero el planteamiento no seajustaalas demandas delaactual sociedad. Las Bibliotecas Escolares no sólo deben contribuir aqueel alumnoaccedaalainformación, como diceel punto 3, sino quelasBibliotecasEscolares han de serelCentrodeReaursosparaelAprendizaje, quedebe estar incluido en el proyecto educativo y en el organigramadel œentro.

${ }^{*}$ En los países donde se ha abordado legislativamente el tema delas Bibliotecas Escolares y éstas se han integrado efectivamente en el sistema educativo y bibliotecario existe un mayor número de Bibliotecas Escolares bien dotadas y con personal especializado, esta situación se observa claramente en Francia, Estados Unidos y Gran Bretaña.

** Entendemos quelas Bibliotecas Escolares no sólo son necesarias en los centros públicos, también deben exigirse en los centros privados unosmínimos. 
El punto 5resultaambiguo pues parecedejarabiertala posibilidad de poder prescindirdeBiblioteca escolar en loscasos en que existauna Biblioteca Pública œercana o enlamismalocalidad. FinalmentelaLOE omitealgunos puntos importantísimos, como son los recursos humanos, las infraestructuras y el modelo deBE, sin embargoglobalmenteesuna regulaciónimportantísima, sin precedentes en losúltimos años, punto departida para un cambio significativo con respecto a la la profesionalización y reconocimiento de la función bibliotecaria en laEscuela, con plenaintegración en los claustros deprofesores

\section{SituaciónactualdelasBibliotecasEscolares}

Las fuentes a nuestro alcance para poder presentarla situación actual delas BE son abundantes. Setrata de estudios realizados en distintas comunidades españolas en los años 90 y principios del 2000 , los resultados que presentan estostrabajosno sealejan significativamente del análisis general delasbibliotecasescolaresen España realizado a instancias del Ministerio deEducación en 1995y $1996 *$ con el fin deemprender un Plan deMejora en el contexto de la reforma educativa socialistay del recienteestudiollevadoacabo porlaFundación Germán Sánchez Ruipérez eIDEA. El estudio, coordinado por ANABADY FESABID, sellevó acabo sobre una muestra total de 750 centros escolares, delos cuales 387 eran de Educación Primariay353deEducación Secundaria. Las autorastoman comoreferenciaparael diseñodel estudio las directrices internacionales elaboradas por la UNESCO en 1979, desarrolladas, más tarde, por la sección deBibliotecas Escolares delaIFLA.

Los aspectos más destacados del análisisllevado a cabo son:

Dotación debibliotecas en los centros escolares; integración delaBE en el centroeducativoyrelación con otras instituciones; recursos documentales ofrecidos; espacios y dotación de equipos; fFuncionamiento, en cuantoalos servicios, aspectos económicos de la gestión, personal, horario de aperturaydel responsabledelaBE.

Las conclusiones del estudio evidencian cualitativamenteunasituación deestancamiento, con

*BARÓ, M. y MAÑÁ, T. (dir.). 1996. Situación delasbibliotecasescolares en España. ANABAD y FESABID. Un resumen de las conclusiones de este estudio puede consultarse en: BARÓ, M. y MAÑÁ, T. La situación de las bibliotecas escolares en España: estudio a nivel nacional. Educación y biblioteca, no 71, pp. 7-16. gran precariedad demediosydematerial, ademásel estado deficitario de las BE no se atiene en absoluto a los parámetros cuantitativosycualitativos quemarcan las directrices internacionales. La BE no se encuentra suficientementeintegrada en el centro educativo, sin proyección en el proyecto curricular y sin reconocimiento por parte del claustro. Es notablela carenciadepersonal adecuado, cualificadoy oon horario claramente insuficiente siendo sustituido por el voluntarismo del profesorado.

En losañosnoventay comienzos del 2000, en distintas comunidadessehan realizadoinvestigaciones dedistinta envergadura sobreel estado delas BE, queseñalamos a continuación:

Enla Comunidad Canaria, en 1998 sellevóa caboel estudio Análisis dela situación delas BE públicas en Canarias*; en Andalucía en 1999 sepublicó el estudio realizado por la Consejería deEducación yCienciaLas BEenAndalucia*; ; EnExtremadura, en2000, FabaPérez llevóacabolainvestigación LasBibliotecasEscolaresen Extremadura: un estudio comparativo nacional e internacional $^{* * *}$; En Cataluña, a instancias del Ayuntamiento de Barcelona, en 2002, se realizó un estudio sobre Bibliotecas de centros públicos de Educación Secundaria en Barcelona ${ }^{\text {*atok }}$; En Murcia, en 2002,J.A. GómezHemándezpublicaLosproblemasdelas BibliotecasEsoolaresdelaregióndeMurciaenuncontextode crisisdel sistemaeducativo ${ }^{\text {sexpex }}$,losresultadosquesedetallan en esteestudio proceden dela investigación llevadaa cabojuntoaValeraEspínen2001,LaBibliotecaEscolaren

* Puede consultarse en: UNIVERSIDAD DE LAS ISLAS DE GRAN CANARIA. 2004. Análisis dela situación delas Bibliotecas Escolares públicas en Canarias. [en línea]. Servicio de Biblioteca y Documentación. Disponibleen: $\measuredangle$ http:// biblioteca.ulpgc.es/directorio/ biblio_canarias/bcanarias.shtml?analis > [Consulta: 21-1-2007]

** La edición impresa del estudio es la siguiente: ANDALUCIA. Dirección General de Evaluación Educativa y Formación del Profesorado. 1999. Las Bibliotecas Escolares en Andalucía. El Sistema Educativoen Andalucía. Curso 97-98. Sevilla: CEE, 1999.

*** El estudio puede consultarse en: FABA PEREZ: C. Las Bibliotecas Escolares en Extremadura: un estudio comparativo nacional e internacional. 2000.Boletín deANABAD. № 50, pp. 119-133.

**o* El estudio está publicado: BARÓ, M, MAÑ́́, T. y COMALAT, M. Las Bibliotecas de los centros públicos de Educación Secundaria en la ciudad de Barcelona. [en línea]. Anales deDocumentación, no 5, 2002. Disponible en: <http:// www.um.es/fccd/anales/ad05/ ad0503.pdf>. [Consulta: 14-1-2007].

rete*t* GÓMEZ HERNÁNDEZ, J. A. 2002b. Los problemas de las Bibliotecas Escolares dela región de Murcia en un contexto de crisis del sistema educativo.[en línea]. Anales de Documentación. oㅡ 5 . Disponible en: <http:// eprints.rclis.org/archive/00002812/01/ ad0507.pdf>. [Consulta: 14-1-2007]

******* Este estudio al que alude J .A. Gómez Hernández permanece inédito. 
la región deMurcia ${ }^{* * k * k *}$, ainstancias dela Dirección General de Formación Profesional e Innovación Educativa delaConsejería deEducacióny Universidad delaRegióndeMurcia; EnelPaísVasco,en2002,sepublioó el estudio Informesobrela situación delas Bibliotecas Escolares en la Comunidad Autónoma del País Vasco* realizado por el Departamento deCulturay Educación; en 2003, la Consejería de Educación y Cultura del PrincipadodeAsturias publicauninformeestadístioo sobrelasituación delasBEen Asturias ** En Castilla-La Mancha, en 2002-2004, desde la Consejería de Educación yCulturaen colaboración con la Facultad de Documentación delaUniversidad deAlcalá serealizó un estudio debibliotecas ubicadas en centros docentes no universitarios, incluidos los Centros deProfesoresy Recursos ${ }^{* * *}$.

El último estudionacional sobrelasituación delas BE: LasbibliotecasescolaresenEspaña: análisisyrecomendaciones hasidorealizadolaFundación Germán SánchezRuipérez (FGSR) eIDEA ${ }^{* * * *}$ duranteel curso 2004-05. Setratade un exhaustivo estudio sobreunamuestra de 401centros públicos y concertados de enseñanza primaria y secundaria, 3.848 profesores y 16.056 alumnos. Los aspectosmás destacados del análisisllevadoa cabo son: existencia o no de biblioteca central, existencia de Bibliotecasdecentro/ bibliotecasdeaula, equipamiento, instalaciones y tecnología, $\mathrm{n}$ o de documentos en la biblioteca central, $\mathrm{n}$ o de documentos / alumno, tratamiento técnico y automatización, personal, dedicación del responsable, horas deapertura, servicios ofrecidos, programasformativos paraprofesores.

Losdiferentesestudios autonómicoslamentablemente presentan resultados semejantesalosmostrados porel estudio deámbito nacional realizado por ANABAD. FESABID ylaFGSR. Entrelas deficiencias detectadas sobresalen los siguientes aspectos: la BE carece de

\footnotetext{
${ }^{*}$ ARREGUI BARANDIARAN, A. 2002. Informesobrela situación de lasBibliotecas Escolaresenla ComunidadAutónoma del PaísVasoo. [en línea]. Disponible en: <http:/ / www.berrikuntza.net/ edukia/ liburutegiak/dokumentuak/informe_bibliesc.doc $>$. [Consulta: 23-12007].

** Puede consultarse el estudio en: ASTURIAS (Comunidad autónoma). Consejería de Educación, Culturay Deporte. 2002. Plan deactuación para el desarrollo delas Bibliotecas Escolares en Asturias. [CD-ROM].

*** Pueden consultarse resultados del estudio en: ORTIZ- REPISO, V.y CAMACHO, J . A. 2005. Radiografía delas Bibliotecas Escolares de Castilla-La Mancha. [en línea]. Educar, no 22.

Disponible en: <http://www.jccm.es/educacion/educar/num_22/ index.html >. [Consulta: 14-1-2007].

MA**** MARCHESI, A., MIRET, I. (Dir.) 2005. Las bibliotecas escolares en España: análisisy recomendaciones. Madrid: FUNDACIÓNGERMÁN SÁNCHEZRUIPÉREZ eIDEA.
}

relevancia en el proyecto curricular del centroeducativo. Losserviciosselimitan alaatención desala delecturay préstamo delibros; el diseñoespacial esinadecuadoyel horario insuficiente; la organización técnica es incipiente, la infraestructura espacial y tecnológica claramenteinsuficientesylos recursos deinformación limitados; laimagen delaBE resultainfravalorada en la jerarquía educativa; el personal a cargo delabiblioteca carece, a menudo, de formación bibliotecaria; la Biblioteca Escolar adolece de un programa formal de promoción ala lectura; el docenteno vinculasu trabajo al uso de los recursos disponibles en la biblioteca y desconoce el uso potencial de las tecnologías y su aplicación pedagógica.

Apesardeestasituación, laBEno hasupuesto un tema prioritario deinterés paralaadministración educativa, locual ponederelievequelosgestoreseducativosignoran la importancia de la BE y no la consideran el espacio necesario para el aprendizajepropio dela Sociedad del Conocimiento.

Tras algunos intentos fallidos de reforma, la BE ha quedadoreducidoal quehacervoluntariodel responsable bibliotecario de los centros*, al ámbito de las Asociaciones, que suelen centrar su actuación en la animaciónlectorayalaactividadinvestigadora, queestá generadointeresantespropuestasy publicaciones.

Ladécadadelos 90 hasupuesto un tiempo degrandes cambios en los modos deproducir información ycrear conocimiento, y las bibliotecas escolares, que como hemos analizado, secaracterizan por su precariedad y marginalidad, no han podido adecuarse a las nuevas necesidadeseducativas. El retraso en la dotaciónypuesta enfuncionamientodeestosserviciosdeinformación para el aprendizajelos marginay losaleja cadavez más dela vidadel centroydelosusuarios, convirtiendoen urgente sunecesidad derenovación.

\section{LA BIBLIOTECA ESCOLAR EN LA SOCIEDAD DEL CONOCIMIENTO: EL CENTRO DE RECURSOS PARA LA ENSEÑANZA Y EL APRENDIZAJE(CREA)}

Laidea tradicional debibliotecacomolugaraisladodela dinámicaesoolar, destinadaa organizaryoonservarlibros yoon un usolimitado sealejaclaramentedel sentidoque las bibliotecas cobran en la Sociedad del Conocimiento.

* Loables esfuerzosindividuales quetienen un carácter más testimonial que riguroso y no han podido extenderse por la falta apoyo y financiación delas administraciones educativas. 
No será posiblequelos ciudadanos alcancen un nivel óptimo de competencias quele permitan participar plenamente en la sociedad sin el concurso detoda la infraestructura cultural, habrá, portanto, quereformular el concepto de Biblioteca, en todos sus ámbitos de actuación, y alentar su papel en la educación y el aprendizaje(Astay Federighi, 2000).

Laprácticadocentecon mediosymaterialescurriculares ha estado organizada tradicionalmente en torno a un tipo concreto de tecnología, el material impreso, en la Escuela hareinado desdesiempreel libro detexto*. Sin embargo, esta hegemoníaincuestionable en tiempos pasados está destinada a experimentar una gran transformación provocada por los cambios que han introducidolas tecnologías, especialmenteInternet.

Los nuevosinstrumentosy herramientas tecnológicas (dispositivos dediferentestipos, ordenadoreseInternet entre otros) queinundan nuestro entorno más cercano también van aumentando su presenciaa la escuela. La práctica más común, hasta el momento, ha sido centralizar la tecnología prioritariamenteen les aulas informáticas, sinembargo, no es el lugar adecuado para el usoeintegración efectiva delas grandes posibilidades didácticas delosrecursoseducativos digitales; pruebade ello es el escaso número deprofesores queapoyan sus clases utilizando el aulainformática. Las posibilidades informativas y comunicativas que presenta Internet podríahacer posibleunaescueladiferente, más cercana ala realidad del alumno, abiertaal mundo, una escuela en la quealumnosy cada profesores puedan accedera materiales didácticos de todo tipo y a un entorno informativo deunariquezasin precedentequefavorezcan laconstruoción deconocimiento. Paraaprovecharestas posibilidades necesitemos infraestructuras complementarías en las propias clasesy especialmente en lasbibliotecas escolares, un medio privilegiado con capacidad paraalbergarrecursosimpresosy digitalesen igualdad decondiciones, en el quelos alumnos puedan continuar sus aprendizajes, iniciar otros nuevos y disfrutardesu ocio.

Laideadeunaaoción formadoraquehademantenersea lolargo delavida ha sustituido, en losúltimosaños, al conceptotradicional deeducación en una etapaconcreta

*En algunos centros con metodología didáctica propia, como el Colegio Estudio de Madrid, inspirado en los principios educativos de La ILE, se ha procurado desdesus inicios aplicar una filosofía de educación a los alumnos en el uso dela información, enseñándoles a seleccionary organizar la información mediante un elaborado trabajo manual a base defichas. delavida. Para conseguir ese objetivo decontinuidad habrá queunir, poruna parte, las políticas educativasy porotrolasvíasnooficiales deaprendizaje. Enestaunión lasnuevas BibliotecasEscolarespueden desempeñarun papel importantísimo, queno hadelimitarseaunamera interacción con el sistema escolar. Deben ser centros dinámicos, con un nuevo espacio-entorno y un innovador conœepto deservicios queacerquelaEscuela al mundo real, quela enriquezca y la haga accesiblea todoslos ciudadanos, favoreciendolaigualdad.

El nuevo modelo de Biblioteca que presentamos, el CREA, conjuga los elementos tradicionales delas BE con la necesaria dimensión educativa y formadora, propia delaSociedad del Conocimiento. Contemplamos, además, la plenaintegración de las Tecnologías dela Información y Comunicación, para hacer posiblela formación alolargo delavidael aprendizajeautónomoy laAlfabetización en Información detodoslosciudadanos.

\section{ElmodeloCREA:marooteónioo}

La evolución delaBE, desdesu concepción tradicional, al modeloqueproponemos, el CREA, parte, en rigor, de lanecesidad deuna revisión teórica por los efectos que los cambios educativos, sociales y tecnológicos han impuestoalaEducaciónylas deficiencias detectadasen lasituación actual delas BE denuestro país.

El conceptoclásico deBiblioteca resultainsuficientepara dar cuenta de los cambios que esta institución está experimentando, es por eso quelas tres propuestas teóricasqueentendemoscomo antecedentes denuestro modelopresentan modificaciones enladenominación.

LaBibliotecaCentrodeDocumentación(enadelante BCD), modelo francés. En 1984 el Ministerio de Educación Nacional y el Ministerio de Cultura de Francia, promovieron unaaoción experimental ${ }^{*}$ conjunta para la promoción de la cultura y del libro, que acompañada por la publicación deun documento en el que se definía el papel dela biblioteca en los centros educativos ${ }^{*}$, sirvió como impulso definitivo para el nacimiento de las BCD, en los centros de educación primariaylos Centros deDocumentacióneInformación (enadelanteCDI) parasecundaria(collègesylycées). El

\footnotetext{
*Acción experimental realizada en los distritos educativos de Créteil, Grenoble, Lyon, y Poitiers, para impulsar el desarrollo delas BCD en los centros educativos

${ }^{*}$ Circularno 84-360, de 1 de octubre de 1984, que expresa el acuerdo entrelos Ministerios de Educación Nacional y Cultura de Francia en el ámbito dela lectura.
} 
nuevo concepto hace alusión a un tipo de BE muy diferenteal tradicional, tanto en su estructura como en sufuncionamiento. LaBCDoCDI ocupaunlugarcentral en el centro educativo, inmersaen el proyecto curricular de de los centros. El espacio está abierto y accesible permanentemente, losalumnos pueden acudirsoloso acompañadosporsusprofesoresypuedeelegirlibremente sus actividades o lecturas, sin la intervención deun adulto. Estas bibliotecas acogen diferentes tipos de actividadespara su dinamización: lecturas, audiciones, préstamos, clubdelectura, consulta deinformación para laindagación y conferencias entreotras. Están abiertas, asímismo, alacolaboración depersonas externas: padres, escritoresy profesionales del libro, entreotros. Las BCD francesas han intentado liberaralalecturadesu estatus de actividad puramente escolar para otorgarle su verdadero papel social, al mismo tiempo queserecupera laideafundamental del niño como protagonista desus propios aprendizajes. Portanto, laBCD francesaintenta modificarel conjuntodelasrelacionespedagógicas(J ordi, 1998) ysuponen un pasoimportantísimo enlaevolución de las Bibliotecas Escolares hacia la Sociedad del Conocimiento, sin embargo al pretender que su estructurasea autogestionadaporlosusuarios, queson principalmente alumnos y profesores, soslaya la importantísima tarea técnica y formadora del bibliotecario documentalista.

\section{ElCentrode recursosmultimediales oCentro de RecursosparaelAprendizaje(CRA).Modeloenunciado} en Hispanoamérica, difiereen sufuncionalidad respecto a los nuevos modelos bibliotecarios que se está implantandoen algunos países deEuropa. El Centro de Recursos Multimediales* es el espacio de carácter pedagógico quecontribuyedemanera permanenteala innovación educativa. Reúne una variada gama de recursos de información en diversos soportes (bibliográficos, electrónicos, yaudiovisuales, entreotros) organizándolos en función a los requerimientos del proyecto curnicular del œentroponiéndolosadisposición delos usuarios, alumnos y profesores, a través desus variados servicios. Es notable el gran esfuerzo institucional realizado por países como Uruguay, Perú, Paraguay $^{* *}$, Venezuelay, sobretodo, Chile. En Chilese

\footnotetext{
* Según define PÉREZVELA, E. 2000. Debiblioteca escolar a centro de recursos multimediales : Construyendo un modelo. [en línea]. Biblios, año 2, no 5. Disponibleen: বhttp:// www.documentalistas.com/ web/ biblios/articulos/5/ B5-03.pdf>. [Consulta: 14-1-2007].

** Puedeconsultarsela página del Ministerio deEducación Paraguayo sobre los CRA. Disponible en: <http:// www.educacion.gob.pa/ cra.htm>. [Consulta: 14-1-2007].

*** El Ministerio deEducación deChilemantiene portal deapoyo alos CRA. Disponible en: <http:// www2.mineduc.cl/ cra/ index.php>. [Consulta: 14-1-2007].
}

estállevando a cabo una gran reforma educativa desde mediado de los años 90 con una incidencia importantísima en las $\mathrm{BE}^{* * *}$, quevan siendo dotadas paulatinamenteconrecursoseconómioosyhumanospara su completa renovación y conversión en Centros de Recursos para el aprendizaje. Lafunción principal del CRA en el liceo o escuelas de Chile* es apoyar la implementación del currículum, actuando como un espaciogeneradordeinstancias deaprendizaje. Através delos CRA seintegra efectivamentelabiblioteca oon el curriculum escolar, sefacilita información y difusión a profesores y alumnos sobre los recursos y materiales disponiblesy se potencialabiblioteca como un lugarde encuentro, intercambioy cooperación.

El CRAI (Centros de Recursos parael Aprendizajeyla Investigación). El nuevo concepto de Biblioteca Universitaria, concebido en el Espacio Europeo de Educación Superior (en adelanteEEES) a partir dela Declaración de Bolonia se estáimplantando con gran rapidezylas BibliotecasUniversitarias (en adelanteBU) están viviendo un interesante proceso de renovación paraadaptar susfuncionesy serviciosala Universidad que debe surgir de la aplicación del proceso de convergencia que auspicia el EEES. Esteproceso de cambio suponelanecesidad dequelasBUtransiten hacia el nuevo modelo de biblioteca denominado CRAI. Un CRAI, según define REBIUN, ,* esun entorno dinámico en el que seintegran todos los servicios universitarios que dan servicio al aprendizaje y la investigación,

\footnotetext{
${ }^{*}$ La reforma curricularchilena proponequeel aprendizajedebelograrse en una forma de trabajo pedagógico, quetiene por centro la actividad delos alumnos, sus características, y sus conocimientosy experiencias previas. El CRA, en este contexto, es fundamental.

** REBIUN (Red de Bibliotecas Universitarias), organismo quereúne a las bibliotecas universitarias españolas, está impulsando la investigación y la transformación de éstas en CRAI, con el fin de proporcionar el apoyo adecuado al modelo educativo universitario europeo. Pueden consultarse sus documentos, publicados en la web deREBIUN. Disponibleen: 〈http:/ / bibliotecnica.upc.es/Rebiun/nova/ principal/index.asp>. [Consulta: 14-7-2006].

*** En torno a los CRAI comienzan a gestarse proyectos de investigación, grupos deinvestigación y experiencias. Como ejemplos enoontramosel Proyecto: Dela Biblioteca Universitaria al Centrode ReaursosparaelAprendizajeeInvestigación.Elaboracióndeunaguía sobrela organizacióny gestión deun CRAI en el contextodelas universidades españolas. Proyectoyafinalizado(Febrero2004-Octubre 2004), coordinado por el Dr. Area, dela Universidad dela Lagunay en el que ha participado el Dr. Marzal García-Quismondo, director de esta tesisy su equipo. Otro proyecto, que aborda el tema, se está desamollandoenlaactualidadesDocumentacióny tecnologíasdela informaciónparaEducación: herramientasparalaalfabetización informacionaly organizaciónderecursos didáctioos(Subdireoción General deProyecto deInvestigación, Secretaría de Estado dePolítica Científica y Tecnológica, Dirección General de Investigación, Ministerio deCienciay Tecnología (2003-06) dela Universidad Carlos III de Madrid, coordinado por el Dr. Marzal García-Quismondo. En ambos proyectos participo como investigadora colaboradora.
} 
vinculadoscon el mundo delainformaciónylas Nuevas Tecnologías. Entendemosqueel CREA, porsunaturaleza y objetivos, se acerca más al modelo CRAI ${ }^{* * *}$ que a cualquierotro modelobibliotecarioy ha deinspirarseen él en su proceso de transformación. Losnuevos Centros deRecursos han deatender las demandas delasociedad en su integridad, no deben limitarsea una etapa dela vida, la universitaria por ejemplo, ni aun solo sector de lapoblación.Lanecesidad deinformación, el aprendizaje, lalectura, seinician enlaEscuela, losCREA, queasumen función deBibliotecaEducativa, portantoson necesarios desde la etapa escolar, reafirmando el principio de igualdad del derecho alaeducación, laculturayel aoceso alainformación.

\section{Definición deCREA:}

Entrelagran variedad derasgosquepueden caracterizar a un CREA proponemos unos puntos centrales, que valoramos comocaminos paradefinir su futuro:

Lacapacidad paraorganizaryrepresentar el conocimiento expresado y difundido mediante diferentes tipos de documento educativo; la capacidad para organizar, gestionar y hacer posible el acceso a los recursos de información; laaptitud paraorientar, formareinformar ala comunidad educativa con personal especializado; la capacidad para promocionar lalectura, en sus distintas dimensionesylaposibilidad desustentarunnuevomodo deconocera través delaAlfabetización en Información.

El CREA enriquece la noción tradicional de BE al definirsecomo un nuevo espacio educativo dinámico, nomerogestorderecursoseducativos, sinoámbitopara una metodología didáctica activa, interdisciplinar y adaptadaaladiversidad deentomosyaprendizajes, œentro suministrador, organizador desaberesypotenciador del autoaprendizaje, no complemento del currículum académico, sino parte integrantedeél. Un espacioentorno para la formación, la información, el entretenimiento, el intercambioyel conocimiento.

\section{Aspectostécnicosyorganizativos: Nuevodiseñode espaciosysenvicios}

El CREAhadeteneruna autonomíafuncional similara ladecualquier departamento del centro educativo, con supersonal adscrito, capacidad degestión, programación, evaluacióny control.

Alproponer laslíneasparalatransformación tecnológica y procedimental de la BE en CREA, como servicio documental y educativo, debemos considerar la interrelación entre los componentes tecnológicos, educativos e informativos de la biblioteca y los componentesorganizativosyespacialesdelainstitución, decuyacombinación surgeel nuevo concepto deCREA.

\section{Nuevasnecesidades:procedimientos}

El CREA no anula las funciones tradicionales de biblioteca sino que las enriquece con la asunción de nuevas atribuciones queseañaden a las ya existentes, comoson:

Servir como soporte ala enseñanza, desarrollando la autonomía personal en los propios procesos de aprendizajeatravésdelaAlfabetización en Información; hacer posible el blended learning, aprendizaje que incrementalas posibilidades dela presencialidad con el aditivodel aprendizajevirtual; serunlugar deencuentro cultural y creativo. Espacio socializador en el quelos usuariostengan laposibilidaddedesarrollaractividades que atiendan tanto a favorecer sus hábitos de comunicaciónydelecturacomoamejorarsusniveles de autonomía en la adquisición de conocimientos que cubran sus necesidades formativas, informativas y lúdicas; favorecer la puesta en funcionamiento y el desarrollo decomunidades virtuales deaprendizaje; potenciar la producción de contenidos educativos; facilitar laorganizacióny aoceso alos recursos digitales.

El espacio en el CREA lo abondamos desdeel punto de vista del servicio que ha de ofrecersea un público muy puntual, la comunidad educativa, en un contexto definido, laSociedad del Conocimiento. No setratasolo del espacio interno dela biblioteca, sus documentosy recursos. Eselugar hasido tradicionalmentemixto: las salas, lasestanterías, el depósito, lasmesas, los expositores y la colección. Actualmente el enfoque espacial es igualmenteválido, pero su complejidad seincrementa debido a la trascendencia que adquieren los espacios electrónicos de acceso a lainformación einteracción con el usuario, convirtiéndose en un espacio-entorno representado por lainformación digital, las redes de comunicaciones y el espacio de interacción. La importancia queadquiereel entorno en el contexto del CREA hacenecesario un nuevo planteamiento espacial en el quehan detener cabida, además delos elementos tradicionales, los siguientesespacios:

Zonadeordenadores con acceso a Internet; laboratorio deidiomasymultimedia; áreadefacilidadestecnológicas: 
edición-impresión; áreadetrabajo en grupo; áreacultural yrecreativa.

Además, integrando los servicios clásicos con los más innovadores deben incorporarse los siguientes elementos:

Catálogo automatizado; depósitodemateriales docentes: Apuntes, modelosdeexámenes, ilustraciones prácticas; repositoriodeObjetos deaprendizaje(learningobjects); depósito de archivos gráficos; depósitos de libros electrónicos (E-book) y revistas electrónicas; servicio depréstamo einformación on-line; organización dela información electrónica producida en la institución, servicios desoportey asesoramiento tecnológico a la comunidad educativa. secursos humanos profesionales; videoteca digital, puntos deacceso a Internet en todas lasplazas delectura.

\section{Organización delconocimientoparaelaprendizaje significativo: OontribucióndelaDocumentaciónpara lagestióndecontenidosenelCREA}

Unadelastareasfundamentales delAnálisisDocumental de Contenido, para hacer frentea los retos impuestos porel salto delaSociedad delaInformaciónala Sociedad del Conocimiento, esactuarcomo soportealos nuevos modelos de bibliotecas. Al haberse generado nuevas necesidades sehan ido articulando modelos teórioose instrumentos que deben resolver los problemas detectados. Esta situación se hace especialmente evidente en un ámbito dondelos contenidos son de especial trasœendencia, la Educación. Estos modelose instrumentos, según apuntan las investigaciones de Marzal, Cuevasy Colmenero (2005a), presentan unos campos deactuación prioritarios quetratan deresolver el problema deasociatividad quepresenta el contenido delosrecursos digitales deinformaciónyal desarrollo de instrumentos parala representación, recuperacióny óptimavisualización deestosrecursos.

Lo digital es un aspecto del mundo real y no debemos entenderloaislado del contexto organizativo en el que sedesarrolla, sobretodo si tenemos en cuenta quegran partedelainformación relevanteprobablementeseguirá circulando en formatos no digitales o no integrados (Chowdhury, 1999), por eso es un gran desafío la integración de recursos electrónicos con las funciones tradicionales delabiblioteca, queha deconseguirseen los CREA.

UnCREA debemantener losserviciosqueleson propios como bibliotecaen cuantoadepósito, coleociónyproceso técnico, funcionando, además, con respectoala gestión delos recursos, comolas Bibliotecas Híbridas, pensadas, según sostiene Rusbridge(1998), para armonizar las tecnologías en el contexto de una biblioteca en funcionamiento, al tiempo que para explorar la integración de sistemas y servicios en contextos electróniooseimpresos. En estesentido el CREA habrá deincluir el tratamiento y acceso a distintos tipos de recursos:

Recursos tradicionales: reaursosnodigitalizados, en distintosformatos: libros, mapas, documentosen soporte magnétioo, etc.

Recursos detransformación: recursostradicionales digitalizados para su conservación u óptima visualización.

Recursos digitales: diseñadosespecíficamenteparael entorno digital, con distintos formatos, tecnologías y sistemasdeacoeso.

Recursosvirtualeseducativosnormalizadosuobjetos deaprendizaje: en estetipo derecursos, laformade representación através demetadatosylasposibilidades derecuperación forman una unidad. Los objetos de aprendizaje, como recurso educativo en su dimensión documental, representan actualmente una de las contribuciones más poderosas paralos CREA.

\section{LosreaursoshumanosenelCREA}

Enlaactualidad puedeparecerinnecesarioincidiren la importanciaqueen todaorganizacióntienenlosrecursos humanos, de esta situación da cuenta la abundante bibliografía que en los últimos años se ha venido publicando sobre el tema y la atención que le han dedicadolos OrganismosBibliotecariosIntemacionales*. El desarrollo constante delas técnicas de gestión, el propio proceso dela globalización y con ello el aumento delacompetitividad han convertido, cada vezcon más fuerza, alos recursos humanos en el mayor elemento estratégico decualquier organización (Armario, 2001).

Ahorabien, estaimportancia estratégica no siemprese havaloradodelamismaformay, muchasorganizaciones no le han otorgado la suficienteatención, es el caso de lasBibliotecas Escolares, tradicionalmenteatendidaspor

${ }^{*}$ Como ejemplos significativos podemos aportar el informepreparado parala DGXIII/E/4, dela Comisión Europea sobre el estudio de Las bibliotecas públicasy la Sociedad dela Información: IFLA/UNESCO. 2001. ThePublicLibrary Serviœe: IFLA/UNESCOguidelinesfordevelopment. Munich: Saur, XVI. 
personal, aunquevoluntarioso, escasamentecualificado. Paralograr losobjetivos previstos en el modelo CREA, ser un servicio global e integrado de información, formacióny orientación, y asegurar una gestión eficazy una proyeoción ooherentedel Centro de Recursos hacia la comunidad educativa, es absolutamentenecesario contar con los recursos humanos adecuados. El CREA vaanecesitar parasuóptimo funcionamiento un equipo multidisciplinar, formado por profesionales de la Pedagogía, la Informáticosyla Documentación, delos que, al menos el profesional documentalista ha de trabajaratiempocompleto.

El papel de los bibliotecarios-documentalistas ha cambiado en los últimosañosindependientementede que su lugar de trabajo sea una biblioteca pública, especializada, universitaria, un centrodedocumentación oun servicio deinformación deempresa(SerrayCeña, 2004). El entorno, las herramientas y los recursos de información han evolucionadoy sehan incrementadoy es previsible que seguirán haciéndolo, por tanto, consideramosnecesariointroducirnuevascompetencias alasya tradicionales en estos profesionales en un clima deformación continuay plenaactualización. Frenteal especialista perfectamente cualificado en una técnica concreta es preferible un profesional con formación científica pero con tendencias polifacéticas, abiertoala formación permanente, que posea las competencias necesariasparapermitirlehabilidadesennuevastécnicas.

En el contexto del CREAlascompetencias profesionales del personal han de atender a funciones técnicoorganizativas, asesoramiento, formación, promoción a lalectura, alfabetización en Información.

Las competencias genénicas necesariasparacumplircon su tareaserán:

Conocimiento dela organización a la queperteneceel CREA, capacidad yvoluntad deaprendizajecontinuo, capacidad paratrabajarengrupo eintegración en equipos multidisciplinares, habilidadescomunicativas, habilidad para compartir conocimiento, capacidad ética en el ejercicio delaprofesión, en el uso delainformaciónyen larelación con laspersonas.

Somos conscientes de que un modelo de recursos humanos como el descrito requiere una dotación económica, especialmenteen el apartado depersonal, que no es fácilmente asumible, no obstante, consideramosindispensableinsistiren queel CREA para funcionar como tal, necesita personal especializadoyla figura del bibliotecarioes, por tanto, indispensable.
Unaalternativainteresante para solucionar la falta de medios económicos suficientes para dotar a todos los centros deun equipo multidisciplinar seńa englobarlas bibliotecas delos centros bajo una red de Centros de recursos para el Aprendizaje. Un determinado CREA podría dar servicio a varias bibliotecas de centros escolares, que habría de tener al frente a un documentalista a tiempo completo. EL equipo multidisciplinardel CREAasumináalafunción deapoyar las necesidades delas bibliotecas adscritas. Y todos los CREA, a su vez, serían coordinadosy apoyados por las unidades educativas correspondientes. Esto permitiría aunar esfuerzos, una mejor gestión de los recursos disponiblesyel desarrollo deinvestigaciones orientadas al desarrollo de métodos de organización del conocimientoadaptados específicamenteal proceso de aprendizaje.

\section{BIBLIOTECAESCOLAR ENLASOCIEDADDEL CONOCIMIENTO: BIBLIOTECA DIGITAL EDUCATIVA}

Del mismo modo quela propia escuela, la biblioteca escolarestádeslocalizada. Esbien cierto quelabiblioteca escolar, según la conocemos (o la imaginamos) debe alcanzarun desarrolloprogramático quesólo apuntaen el mejor delos casos: la coleoción debeestar clasificada conformealas grandesáreas deconocimientohumanoy organizada conforme a sistemas deidentificación y control, quelehagan compatiblecon otras coleociones bibliotecarias; debeser soportedeactividades didácticas innovadoras y reformas docentes, archivo de la producción educativa del centro, pilar del diseño programático y curricular del centro; núcleo de cooperación informativa con una red debibliotecas asimilables a susfines educativos; pulmón deacciones de extensión cultural, animación, dinamización y promoción lecto-escritora, proyectos educativos interœentroso internacionales; espacio paraadquisición dehabilidades metodológicas docenteseinvestigadoras novedosas; laboratoriodel saber, tallerparaunaeducación en técnicas dedocumentación, medianteunarigurosa formación deusuarios, prefacio para el uso diestro de bibliotecasmássofisticadasen su funcionamiento; centro deincorporación detecnologías delainformación (en adelante, TIC), mediante el desarrollo de programas informátioos de gestión dela colección (ABIES ) y aula extendida para préstamos y cooperación interbibliotecaria.

*ABIES es una aplicación informática para la gestión electrónica de
bibliotecas escolares en España, editada por el Ministerio de Educación. 
Laslíneas dedesarrollo arribaapuntadasno pretenden presentarunaparadojaentrelarealidady fantasíadelas bibliotecas escolares en España, sino una sinopsis dela presenciafáctica delabiblioteca escolar en la escuela. No caigamos en el victimismo intelectual, desdén institucional ydesesperación profesional. Labiblioteca escolar ha cobradopresenciay hamotivado unaseriede aocioneseducativas, mientrasasociacionesprofesionales documentalistas, grupos de investigación de muy diferentesáreas deconocimiento, institucionespúblicas y privadasimpulsan un proyecto parabibliotecaescolar. ¿Cuál esel problemadel queadolecen tantasiniciativas? Suadscripción aun ectoplasma educativoeinstitucional, capazdedespertarpasionesliterarias(recordemossueterna mimesis con el Departamento de Lenguay Literatura) desdeel voluntarismoylos gestos heroicosen defensade un modelo cultural basado en la palabra escrita, un movimientopropio, portanto, degenteslibrescasdeletras, dique de contención frente a una rampante cultura iletrada.

Labiblioteca escolar, en estas circunstancias, recibeun cometido cultural y sele concedeuna responsabilidad educadora absolutamente desproporcionados a su reconocimiento institucional y función educativa regulada. La biblioteca escolar, en nuestra opinión, ha ido acumulando disfunciones que han evitado su desarrolloymaduración:

a) Un carácter educativo periférioo, por el propio modelo deEducación. Un modelo educativo que sebasa en el desarrollo del educando en destrezas y habilidades elaborativas (talleres de prácticas) eintelectivas (aula deasimilaciones conceptuales), apenas precisa otros elementosauxiliaresalos programados por el profesor paracada materia concreta. Comotallerdeprácticas, la bibliotecaescolarpasaaserel laboratoriodeanimacióny dinamización de la lectura, sala auxiliar para familianizarsecon unincipientemétodocientíficomediante la elaboración de trabajos encomendados, así como complemento deactividades extraescolares mediante efemérides o deberes (los solucionarios delas ciencias experimentales), incluso de excelentes ensayos de innovación docente, excepcionalespornotenercorrelato con una programación acordeal sistema educativo al uso. Son experimentosimpresionantes del buen docente, son actividadesindividuales del buen alumno.

b) Un carácter esencial y existencialmente biblioteconómiø, dependientedesureconocimientopor unsistemabibliotecario refractario, en unacooperación tan agobiantecomo escasamentesimbiótica por parte delas bibliotecas públicas desdesu vocación educativa.
La biblioteca escolar ha girado su transformación y desarrollodemaduración en tomoala organización del espacio, el desarrollo dela colección y la dimensiónyla calidad de fondos, las más adecuadas técnicas de clasificación, catalogación yordenación documentales, la eficacia en los sistemas debúsquedas, la eficiencia en los serviciosdeorientación yreferenciaentendidoscomo formación de usuarios, la extensión bibliotecaria, un anhelo entusiasta por la gestión automatizada delos servicios (ABIES). Donde se ha logrado una mayor transformación, el carácter paranoico del desarrollo se ha agudizado: tanto más desarrollada la función biblioteconómica, másanémicalafunción escolar,

c) Unaplenaidentificación conlaEnseñanza, un carácter subsidiario para el Aprendizaje, un divorcio con la Educación. Labibliotecaescolar hatendidoaquesumodo deorganización documental seadapteala disposición por áreas deconocimiento curriculares y a los tipos documentales según las convenciones programáticas, convirtiéndose necesariamente en un centro de profundización en la instrucción de saberes correspondientes a disciplinas. Con dificultad y por experienciasindividuales docentes, labiblioteca escolar se contempla, como un espacio para la diversidad curricular. Por el propio modelo educativo, labiblioteca escolarno ha explorado su conversión en un espacio de Aprendizaje, dondese ejerciten las estrategias para el aprendizajealolargodelavida.Paraesteobjetivonobastan, en modo alguno, actividades de fomento para determinadas técnicas (lectura, escritura, metódicas, etc.), sino disponer labiblioteca por tópicos deinterés conformea unos objetivos competenciales, definidos comofilosofía educativa del centroformativo. Porúltimo, exactamenteen paraleloalaescuela, labiblioteca escolar poco tiene que ver con la Educación entendida en su sentido de transmisión y asimilación de valores formativos parasu ejercicio provechoso en el contexto social: el alumno seeduca en el ciberespacio, aprendeenel entorno, sele "enseña" en la escuela.

d) Unaidentificación, aún más acusada quela propia escuela, conla cultura escrita. Desdeel modelo escolar medieval, el progreso civilizador se ha entendido absolutamenteligado al conocimiento manifestado en la palabra escrita, único medio para la expresión dela lógicaylarazón, en oposición desuperación alacultura oral. Una biblioteca escolar asimilada en exceso a la palabra escrita estárenunciando expresamentea otras posibilidades educativas deprimera magnitud. Cuando desde comienzos del actual milenio se abre paso el conoepto dealfabetización eninformación, el problemase enunciabajo otrosparámetros. 
Estas disfunciones se han ido haciendo explícitas e implícitas pero, es nuestra opinión, los análisis y respuestashansidomásreactivosqueproactivos. El frente de acción reivindicativa se ha planteado hacia las instituciones educativas pertinentes, como no podía ser deotro modo, espoleadas por el Espacio Europeo de Educación Superior: las bibliotecasuniversitarias han iniciado una transformación a CRAI (Centro de Recursos para el Aprendizajeyla Investigación). Las reformas educativaspreuniversitarias, muyal contrario, no tienen un estímulo académico tan potente, si bien la presión social es muyfuerteyhaidocreciendo: el Informe PISA* hasido un auténtico revulsivo en el fomento dela lectura. El monocultivonuncaesun factor dedesarrollo, sobretodocuando en tornoalaalfabetizaciónylalectura comienzan a pergeñarse tantas dimensiones conceptuales y funcionales. Lalectura está pasando, claramente, desdefundamentarsesobreuna técnicaa desarrollarsesobreun estímulo: el objetivoya no es sólo lalecturafácilygustosa, imprescindibleantesalaparauna lectura analítica comprensiva, que haga mucho más eficaceslasinferenciasdelainformaciónal conocimiento, el objetivoesalcanzarlacompetenciaenunaalfabetización en constelación, esto es, una competencialecto-escritora diversificada en códigos y espacios, en la que la alfabetización sehatransformadoen unaalfabetización en información, en indistinta, coetánea o sucesiva cooperación con alfabetizaciones bibliotecaria, digital, icónica, multimedia, tecnológica, visual.. Las TICyla webimponían su propiarealidad. Susrepercusiones son bien conocidas y huelga hacer aquí una descripción prolija, alejada de los fines del artículo. Conviene, sin embargo, destacar algunas repercusionesinmediatas especialmenterelacionadas conlasbibliotecas escolares:

El equipamiento tecnológico convertido, él mismo, en recurso educativo, como avalan las experiencias deuso didáctico delos equipos audiovisuales o el desarrollo de laEAO(EnseñanzaAsistidaporOrdenador), derivando haciala visualización dela web por ordenadores con conexión Intemetylapizarra electrónica. Losproblemas queplanteason claros: identidad entremedioymensaje educativo, larupturadelarelación dialógicaen el proceso educativo, ladesvirtuación delafunción magistral (muy relevante en Educación, relativa en Enseñanza), la creencia de que el dominio hábil del procedimiento implicalageneración competentedeconocimiento. El Aula Informática seconvierteen competidor biológico inmiserioondedelabibliotecaesoolar.

\footnotetext{
* Programa internacional de evaluación de alumnos. Disponible información sobre sus actividades y resultados en: <http:// www.pisa.oecd.org> [Consulta: 30/01/07].
}

Ladiversificaciónyrapidísimaevolución delosmateriales y medios didáctioos, definidos en su versión tradicional comoobjetospedagógicostransmisoresdecontenidosy conocimientosquedebenadquirirlosalumnosensituaciones concretas deenseñanza, yen su versión digital como cualquierreaursotecnológiooquearticulaenundeterminado sistema desímbolos ciertos mensajes con propósitos instructivos*. En rápidasucesión losmediosymateriales didácticossehan clasificadoen manipulativos, impresos, audiovisualesydigitales, éstosúltimosen unadimensión hipermedia, que les ha permitido manifestarse en material distribuido porla weby en sitios web educativos. Los problemasquesuscitan son evidentes: su captación formal, selección decontenidos por intencionalidady eficacia educativas, tipificación por niveles cognitivos deconsulta, adaptación aobjetivos didácticosconcretos, navegación hipertextual comprensiva yasimilable.

La posible expansión del libro electrónico, con sus formatos especiales, si bien la verdadera dimensión renovadoraessu acepción en tanto quelibro digital, esto es, aquel queseleería desdeel índicedigital a partir de palabras clave coincidentes con el interés del usuario, disponiéndose el texto conforme a la extracción de párrafos incluyentes de la palabra clave. El reto principalísimo eslarupturadel continuum narrativo, la exigencia de una pericia en lectura digital, pero sobre todo el desarrollo deinstrumentos para procesar el lenguajenatural delosdocumentoselectrónioos: enestos documentos la forma y el fondo se funden en la manifestación decontenidos conceptuales, por lo quela propiaimagen del documento genera conocimiento. La organización dela colección de la biblioteca escolar reclamalaorganización decontenidosdela colección de labibliotecaescolar.

Los contenidos de la información de documentos digitales, en efecto, se transforman en la verdadera frontera delos servicios deinformación educativa. Un problemaverdaderamenteimportanteesquelainmensa mayoníadelosrecursos deinformación educativa ocon intención educativa disponen de contenidos difícilmenteregistrados para generarconocimiento. № es extraño, pues, lanecesidad imperiosa deestablecer unos requisitos queacrediten la solvencia oognoscitiva delos contenidos digitales. pincipios de autoridad, materia, anotación o descripción. El paso dentro delos materialesdidácticoshacialosobjetosdeaprendizajeesun retoinsoslayableparalabiblioteca escolar.

\footnotetext{
** Esta definición y la evolución deestos medios y materiales presenta con precisión AREA, M. Los medios y las tecnologías en Educación. Madrid: Pirámide, 2004.
} 
La rápida evolución dela función y disposición delos mediosymateriales didácticos, así comolarelevanciade loscontenidos, hanllevadoaprimerplano deefectividad educativa la edición, esto es, no sólo una lectura comprensiva del documento electrónico por lectura digital, sino la verbalización del saber, para asentarse como conocimiento, mediantela escritura hipermedia. Docentey discentedeben editar su material didáctico, por definición en caso de que se acometa, indefectiblemente, el uso delosobjetos deaprendizaje. El menudeo de plataformas digitales, softwarelibre, la tecnologíawiki ylosweblogs ofrecen unas posibilidades extraondinarias parauninicio suficienteen estaedición.

El entornotecnológioo superaampliamenteel aula, hasta alumbrarel aula extendida, un espacioeducativo quedebe comportarsecomounacomunidad virtual educativa, con losinstrumentosadeauadosparaelaprendizajecolaborativo mediantela edición compartida demateriales, foros de discusión, ciberoteca, tutoración electrónica, interaoción y virtualidad en el proceso educativo, evaluación continua. La extensión bibliotecaria no sustenta adecuadamentela extensión deinformación educativa parael aula extendida.

Todos estos cambios, junto con otros referidos a los servicios de referencia digitales y de gestión de contenidosvirtualesqueahorano hacenal caso, hanido desarmollandolanoción delabiblioteca digital, unanoción alaqueno hapodidosustraersetácticamentelabiblioteca esoolar, quedebecomportarse, sí osí, comounabiblioteca digital educativa.

\section{INSTRUMENTOS DE GESTIÓN DE CONTENIDOS EN BIBLIOTECAS DIGITALES EDUCATIVAS}

El modelo educativo más apropiado haciala Sociedad del conocimiento, sin duda, pareceabocarnos haciael CREA. Entre distintas razones pedagógicas para responder adecuadamentea los nuevos aprendizajes (permanente y colaborativo), existe un argumento incontestable: la preeminencia delascompetencias en Educación.

Son muchas eimportanteslasrepercusionesqueparala escuelaimplica el nuevo modelo educativo: la primacía de los aprendizajes colaborativo y permanente; la implicación corresponsable del educando en su proceso educativo; lacompaginación deespacios educativos con el aula, a su vez ampliada como aula extendida; la alfabetización en el ciberespacio antela horizontalidad e iconismo de los contenidos en la red, así como la transversalidad delos contenidos deintencionalidad educativa. En el caso dela biblioteca escolar, tal como hemos concluido en el anterior apartado, existe una importante propensión hacia el modelo de centro de recursos, unapropensión quereclama, indefectiblemente, nuevosinstrumentosparalaorganizacióny serviciodela coleociónennuevosentomosyparaunanuevacomunidad educativa. Labibliotecaescolary su entomo seconvierte en otro recurso del nuevo centro de recursos. A este respecto, comienzan a pergeñarselas tendencias más visibles para organizar los nuevos œentros:

a)Flexibilidadenlaetiquetaciónsemánticapararepresentary recuperar contenidos. Laasignación de palabras clave, términosydescriptoreses cadavezmásimportantepara lagestión decontenidos digitales, si bien la procedencia de estas etiquetas semánticas desde "lenguajes documentales" está cada día más contestada, por las innegables ventajas delaincorporación del lenguaje natural en los sistemas deinformación. En estesentido, un instrumento idóneo se convierteel concurso delas técnicas deprocesamiento delenguajenatural(PLN), por variosmotivos. El primero, los contenidos digitalesse disponenenel disaursoelectrónio, onigen del génerodigital, con sus propias estrategias cognitivas para redefinir la coherencia y cohesión en el texto*, lo que explica la cooperación oonlaLingüísticaAplicada ylaLingüística de lared**.Segundo,losopacsyotrosmediosdereauperación informativaseverían muyrenovados con el suplemento delas técnicas PLN, cuyo punto de partida está en la Lingüística deCorpus, unamuestradelalenguaalaquese dotadeobjetivosdefinidos, conformealosqueun corpus es etiquetado (evidencia morfosintáctica), parentizado (evidenciadesus constituyentes sintáctioos) yanalizado (evidenciadesusrelacionessintácticas. Tercero, concede unamuyapreciableamigabilidad parael usuarioen el uso delasherramientas derecuperación deinformación, por cuantoel lenguajenatural puedeser, ahora, muycohesivo en la etiquetación delos contenidos por partedel autor, documentalistayusuario, puestoqueel esfuerzodecohesión semántica se hace oculto y, además, otorga grandes posibilidadesdeasociacionessemánticas, muyútilesenla navegación electrónica. El camino queapuntaAdunaAutoFocus $^{* *+k}$ esunailustración perfectamenteaclaratoria.

b)Asociatividadenlosinstrumentosdecontrolsemánticopara análisis de forma y contenido, restando parte de la

${ }^{*}$ POSTEGUILLOGÓMEZ, S. Netlinguistics. Language, Discourseand Ideology in Internet. Castellón: UniversitatJ aumeI, 2003.

** BOLAÑOS MEDINA, A. et al. Análisis del discurso electrónico: convenciones genéricas, lingüísticasy funcionales. Las Palmas deGran Canaria: Universidad, 2004.

${ }^{* * *}$ Existe explicación del instrumento, como una interesante demo. Disponibleen Internet: \http:/ / www.aduna-software.com/ products/ professional_search/autofocus/ overview.view>. [Consulta: 15/01/07]. 
inflexibilidadjerárquica detesauros, encabezamientos de materia o CDU, fomentando al tiempo una horizontalidad acordecon la navegabilidad en red, un estilo sumamente útil que puede ofrecer como instrumento ThinkmapVisual Thesaurus* ${ }^{*}$ AquaBrowser Library, quepuedehallarsecomoimplementación deun opacdebiblioteca en los Estados Unidos**.

c) Navegabilidad desdelosinstrumentos degestión de contenidos, una evolución queha comenzado desdelas taxonomíasy tesauros, haciala implementación que procuran los mapas conceptuales y topic maps. Atomatización y Web han llevado a los mapas conceptuales al medio digital (CmapTools y DigiDocMap, entreotros), iniciandounaconfluenciacon los tesauros, en proceso paralelo deautomatización, por incorporación funcionalidades hipertextuales que permiten integrar, amododeilustración delos conceptos, todo tipo de recursos digitales a través de enlaces, convirtiéndolosen entomosinteractivos. Estedesarrollo haabierto nuevas perspectivas parasu utilización, entre las que seincluyen el desarrollo conceptual desistemas hipertextuales, estructuras denavegación, indizacióny sistemas de recuperación de información, así como modelos deconocimiento ${ }^{* * *}$.

d) Metadatoscomocontrolydescripcióndecontenidosdigitales y desarrollodevocabularios. Losmetadatospuedenllegara ser un lenguaje dedescripción universal, por lo quese han desarrollado esquemas demetadatos, en tanto que conjunto de reglas y elementos que constituyen un modelo demetadatos. Los esquemas permiten definir unasintaxis, pues establecen los elementosy orden en que habrán de disponerseéstos así como el formato de etiquetado o codificado delosmetadatos, yuna semántica, porcuanto ofrecen recomendaciones deuso delos elementos, devocabularios especializadoso acepciones específicas detérminosen determinados dominios ${ }^{* * a+k}$.

* Disponible en: <http:// www.visualthesaurus.com>. [Consulta:15/ 01/07].

** Disponible en: <http:// abingtonlibrary.asub.edu/ aquabrowser>. [Consultado el 15/ 01/07].

*** MARZAL, M.A.; COLMENERO RUIZ, M.J .; CALZADA-PRADO,

J; CUEVASCERVERÓ, A. Mapas conceptualesy presentación gráfica de tesauros: aplicaciones en bibliotecas digitales educativas. II International Conferenœon ConceptMapping. CMC2006.5-8septiembre, San J osé, Costa Rica, 2006.

* MARZAL, M.A.; CALZADA-PRADO, J ; COLMENERO RUIZ, M.J .; CUEVASCERVERÓ, A. Development of a controlled vocabulary for learningobject's functional description in an educational repository. Proceedings of theInternational Conferenceon Dublin CoreandMetadata Applications, DC2006. 3-6 octubre, Manzanillo, Mexico, 2006 pp. 253258; MARZAL, M.A.; CALZADA-PRADO, J ; COLMENERO RUIZ, MJ .; BELTRÁNORENES, P. The construction of Spanish educational repository: advances and future directions of the DOTEINE project. 10thEuropean ConferenœeonDigital LibrariesWorkshopon “LearningObject Repositoriesas Digital Libraries". Alicante, España22 deseptiembre2006. e) Ontologías, aún instrumento complejo pero con muy interesantes potencialidades. En centros derecursos pueden permitir tresfuncionalidades: lacomunicación para comprensión consensuada entre usuarios; la interoperabilidad en el intercambio dedatos; ingeniería desistemaspordesarrollodesoftware.

f) Plataformas digitalespara la formaciónenALFIN, un recurso digital y virtual integrado por objetos de aprendizaje, sistema cooperativo de comunicación e interactividad, capaces de desarrollar esta especial alfabetización. Existen dos principios que deben diseñarse: un sistemaintegradodecategoríasevaluativos sobre las habilidades-destrezas del educando, en interacción y correlato con categorías evaluativos sobre laarquitectura delainformación del recurso; un sistema integrado deindicadores para establecer el impacto y progreso de los educandos por la consecución de la competencia en ALFIN. Lainoculación dela calidady evaluación en este sistema debe ser un objetivo prioritario.

\section{CONCLUSIONES}

Labiblioteca escolar necesita iniciar cuanto antesuna transformación a CREA, cuyos principios, modelose instrumentos derealizaciónyaestánapuntadosypueden comenzara utilizarse prácticamente. Sin embargo, esta utilización no puededeningunamanera realizarsecomo una simple aplicación mecánica, sino que precisa un modelooarquitecturadeaplicación. Estaarquitecturadebe tenercomo principales elementos constitutivos:

- Iniciar la transformación a CREA renovando el modelo educativo y su desarrollo curricular. El modelo detransformación exigeun maroo educativo quesebase en las competencias, el aprendizaje permanente y colaborativoy el aula extendida, un marco en el queel progreso en el proceso educativo requiera una alfabetizacióneninformación, unacompetenciatransversal que mediantela capacidad de seleccionary evaluar contenidos digitales educativos, permita generar con eficacia conocimiento. Sólo una Reforma Educativa acordeconla Sociedad del conocimiento permitiráy hará útil latransformación al CREA.

- Espreciso tener bien asumidalaidea dequeel CREA no hace desaparecer labiblioteca escolar. Labiblioteca escolar permaneceráy debepermanecercon sucoleoción einstrumentos dedesarrollo, organización y control de su colección, sin embargo esta biblioteca escolar seráun recurso más del CREA. El CREAdebe ser un compendio derecursos, oon suspropias coleccionesdocumentalesy 
políticas dedesarrollo de colecciones específicas, con misionesdiferenciadas.

- EL CREA no debe ser sólo un compendio de colecciones documentales en todo tipo de soporte (crecientemente digital y virtual), con documentos capturados, identificados, controladosyorganizadospara serservidos, debeserun centro para editar documentos digitaleseducativosesenciales, losobjetosdeaprendizaje.

Artigo submetido em 18/09/2007eaceito em 19/10/2007.

\section{REFERENCIAS}

ANDALUCIA. (Comunidad Autónoma). Dirección General de Evaluación Educativa y Formación del Profesorado. Las bibliotecas escolares en Andalucía: el sistema educativo en Andalucía: curso 9798. Sevilla: CEE, 1999.

ANTEPROYECTO de ley orgánica de educación. Disponível em: $<\mathrm{h} \mathrm{t} \mathrm{t} \mathrm{p} \mathrm{:} \mathrm{/} \mathrm{/} \mathrm{w} \mathrm{w} \mathrm{w} \mathrm{.} \mathrm{m} \mathrm{e} \mathrm{c} \mathrm{.} \mathrm{e} \mathrm{s} \mathrm{/} \mathrm{f} \mathrm{i} \mathrm{l} \mathrm{e} \mathrm{s} \mathrm{/}$ Anteproyecto_Ley_Organica_Educacion.pdf $>$. Acesso em: 9 jan. 2007.

AREA, M. Losmediosy lastecnologías en educación. Madrid: Pirámide, 2004

ARMARIO SÁNCHEZ, F. Recursos humanos: formación y capacitación. In: ENCUENTRO INTERNACIONAL SOBRE BIBLIOTECAS PÚBLICAS, PERSPECTIVAS EN MÉXICO PARA EL SIGLOXXI , 1., 2001, México. Anales electronicos... Disponível em: বhttp:// www.goethe.de/hn/mex/retro/spb01_1.htm\#A2>. Acesso em: 21jan. 2007.

ARREGUI BARANDIARAN, A. Informe sobre la situación de las bibliotecas escolares en la comunidad autónoma del país Vasco. 2002. Disponível em: <http:// www.berrikuntza.net/ edukia/liburutegiak/ dokumentuak/informe_bibliesc.doc >. Acesso em: 23jan. 2007.

ASTA, G.; FEDERIGHI, P. El públicoy la biblioteca: metodologíaspara la difusión dela lectura. Gijón: Trea, 2000.

ASTURIAS (Comunidad Autónoma). Consejería de Educación, Culturay Deporte. Plan deactuación para el desarrollo delas bibliotecas escolares en Asturias. [S.l.: s.n.], 2002. CD-ROM.

BARÓ, M.; MAÑÁ, T. La situación de las bibliotecas escolares en España: estudio a nivel nacional. Educación y biblioteca, n. 71, p. 7-16., 1997.

; COMALAT, M. Las bibliotecas delos centros públicos de educación secundaria en la ciudad de Barcelona. Anales de Documentación, n. 5, 2002. Disponível em: <http:/ / www.um.es/ focd/ anales/ad05/ad0503.pdf>. Acesso em: 14jan. 2007.

BOLAÑOS MEDINA, A. et al. Análisis del discurso electrónico: convenciones genéricas, lingüísticas y funcionales. Las Palmas de Gran Canaria: Universidad, 2004.

CARROLL, F. Guidelines for school libraries. TheHague: IFLA, 1990.p. 30-34. (IFLA, 128).
;BEILKE,P.F.Guidelinesfor theplanningandorganization of School Library Media Centers. Paris: UNESCO, 1979.

CUEVASCERVERÓ, A Lectura, alfabetizacióneninformacióny biblioteca escolar. Gijón: TREA, 2007.

CHOWDHURY, G. Digital library research: major issues and trends. J ournal of documentation, v. 55, n. 4, p. 409-448, 1999.

ESPANHA. Ley Orgánica deEducación (LOE) 2/ 2006, de3 deMayo, BOE 106, de4 deMayo de2006. Disponível em: বhttp:// www.mec.es/ mecd/gabipren/ documentos/A17158-17207.pdf>. Acesso em: 14 jan. 2007.

.Ley Orgánica 1/ 1990, de3deoctubrede1990, BOEdel 4 deOctubrede1990. Disponível em: বhttp:// www.mec.es/meod/atencion/ educacion/hojas/E_SistemaEduc/e-1-4.htm>. Acesso em: 14jan. 2007.

. Ley Orgánica 10/2002, de23dediciembre, BOEdel 24 de Diciembre de 2002: Exposición demotivos. Disponível em: <http:// www.igsap.map.es/ cia/ dispo/24826.htm>. Acesso em: 14jan. 2007.

. Real decreto 582/ 1989, de 19 demayo, BOE del 31 de mayo de 1989. Disponível em: <http:/ travesia.mcu.es/ documentos/ rd_seb.pdf>. Acesso em: 14jan. 2007.

FABA PEREZ, C. Las bibliotecas escolares en extremadura: un estudio comparativo nacional einternacional. Boletín deANABAD, n. 50, p. 119-133, 2000.

FEDERAÇÃO INTERNACIONAL DAS ASSOCIAÇÕES E INSTITUIÇÕES BIBLIOTECÁRIAS; UNESCO. IFLA/ UNESCO school library manifesto: theschool library in teachingand learningfor all. 2000. Disponível em: <http:// www.ifla.org/VII/s11/pubs/ manifest.htm>. Acesso em: 9jan. 2007.

School library guidelines. 2002. Disponível em: «http:// www.ifla.org/ VII/ s11/ pubs/ sguide02.pdf>. Acesso em: 9 jan. 2007. Puedeconsultarseel documento también en español. Disponível em: \ttp:// www.ifla.org/VII/s11/pubs/ sguide02-s.pdf>. Acesso em: 14jan. 2007.

GÓMEZHERNÁNDEZ, J . A. Los problemas delas bibliotecas escolares dela región de Murcia en un contexto de crisis del sistema educativo. 2002b. Anales de Documentación, n. 5. Disponível em: <http:// eprints.rclis.org/ archive/ 00002812/ 01/ ad0507.pdf>. Acesso em: 14jan. 2007.

HANNESDÓTTIR, S. K. School librarians: guidelines for competency requirements. The Hague: IFLA, 1995.

JORDI, C. Guía práctica dela biblioteca escolar. Madrid: Fundación Germán Sánchez Ruipérez, 1998.

MARCHESI, A.; MIRET, I. (Org.). Las bibliotecasescolares en España: análisis y recomendaciones. Madrid: Fundación Germán Sánchez RuipérezeIdea, 2005.

MARCOSMORA, M. C. Interacción en interfaces derecuperación de información: conceptos, metáforasyvisualización. Gijón: Trea, 2004.

MARZAL GARCÍA-QUISMONDO, M. A; CUEVAS CERVERÓ, A.; COLMENERO RUIZ, M. J . Instrumentos de utilidad en análisis decontenido parala organización de documentos digitales educativos. In: CONGRESO ISKO ESPAÑA, 7., 2005, Barcelona. Anales... [S.l.: s.n.], 2005a. 
. La biblioteca escolar como centro de recursos para el aprendizaje. In: CONGRESO INTERNACIONAL VIRTUAL DE EDUCACIÓN, 5., 2005. Anales... [S.l.]: Universidad de las Islas Baleares y Cibereduca, 2005b. CD-ROM.

etal. Theoonstruction ofa Spanish educational repository: advances and future directions of the DOTEINE project. In: EUROPEAN CONFERENCE ON DIGITAL LIBRARIES WORKSHOP ON LEARNING OBJ ECT REPOSITORIES AS DIGITAL LIBRARIES, 10., 2006, Alicante, España. Anales...Espanha: [s.n.], 2006.

. Development of a controlled vocabulary for learning object's functional description in an educational repository. In: INTERNATIONAL CONFERENCE ON DUBLIN CORE AND METADATAAPPLICATIONS, DC2006, 1, 2006, Manzanillo, Mexico. Proceedings...[S.l.: s.n.], 2006. p. 253-258.

. Mapas conceptualesy presentación gráfica detesauros: aplicaciones en bibliotecas digitales educativas. In: INTERNATIONAL CONFERENCE ON CONCEPT MAPPING. CMC 2006, 2., 2006, San J osé, Costa Rica. Proceedings... [S.l.: s.n.], 2006.
ORTIZ- REPISO, V.; CAMACHO, J . A. Radiografía delas bibliotecas escolares de Castilla-La Mancha. Educar, n. 22, 2005. Disponível em: বhttp:// www.jocm.es/ educacion/educar/num_22/index.html>. Acesso em: 14jan. 2007.

PÉREZ VELA, E. De biblioteca escolar a centro de recursos multimediales: construyendo un modelo. Biblios, ano 2, n. 5, 2000. Disponível em: <http:// www.documentalistas.com/ web/ biblios/ articulos/5/ B5-03.pdf>. Acesso em: 14jan. 2007.

POSTEGUILLO GÓMEZ, S. Netlinguistics: language, discourseand ideology in Internet. Castellón: UniversitatJ aumeI, 2003.

RUSBRIDGE. Towards thehybrid library. D-Lib Magazine,J uly/ Aug. 1998. Disponível em: <http:/ / www.dlib.org/ dlib/july98/ rusbridge / 07rusbridge.html>. Acesso em: 14 jan. 2007.

UNIVERSIDAD DE LAS ISLAS DE GRAN CANARIA. Servicio de Bibliotecay Documentación. Análisis dela situación delas bibliotecas escolares públicas en Canarias. 2004. Disponível em: <http:// biblioteca.ulpgc.es/directorio/biblio_canarias/bcanarias.shtml?analis>. Acesso em: 21jan. 2007. 